\title{
Market Microstructure: \\ A Self-Organizing Map Approach to Investigate Behavior Dynamics under an Evolutionary Environment
}

\author{
Michael Kampouridis ${ }^{1}$, Shu-Heng $\mathrm{Chen}^{2}$, and Edward Tsang ${ }^{1}$ \\ 1 School of Computer Science and Electronic Engineering, University of Essex, \\ Wivenhoe Park, CO4 3SQ, UK \\ 2 AI-Econ Center, Department of Economics, National Cheng Chi University, \\ Taipei, Taiwan 11623
}

\begin{abstract}
This paper extends a previous market microstructure model, which investigated fraction dynamics of trading strategies. Our model consisted of two parts: Genetic Programming, which acted as an inference engine for trading rules, and Self-Organizing Maps (SOM), which was used for clustering the above rules into trading strategy types. However, for the purposes of the experiments of our previous work, we needed to assume that SOM maps, and thus trading strategy types, remained the same over time. Nevertheless, this assumption could be considered as strict, and even unrealistic. In this paper, we relax this assumption. This offers a significant extension to our model, because it makes it more realistic. In addition, this extension allows us to investigate the dynamics of market behavior. We are interested in examining whether financial markets' behavior is non-stationary, because this implies that strategies from the past cannot be applied to future time periods, unless they have co-evolved with the market. The results on four empirical financial markets show that the markets' behavior constantly changes; thus, agents' trading strategies need to continuously adapt to the changes taking place in the market, in order to remain effective.
\end{abstract}

Key words: Genetic Programming, Self-Organizing Maps, Market Microstructure, Market Behavior

\section{Introduction}

There are several types of models in the agent-based financial markets literature. One way of categorizing them is to divide them into the $N$-type models and the autonomous agent models (Chen et al, 2010). The former type of models focuses on the mesoscopic level of markets, by allowing agents to choose among different types of trading strategies. A typical example is the fundamentalistchartist model. Agents in this model are presented with these two strategy types and at any given time they have to choose between these two. A typical area of 
investigation of these models is fraction dynamics, i.e., how the fractions of the different strategy types change over time. However, what is not presented in most of these models is novelty-discovering agents. For instance, in the fundamentalistchartists example, agents can only choose between these two types; they cannot create new strategies that do not fall into either of these types. On the other hand, the autonomous agent models overcome this problem by focusing on the microscopic level of the markets. By using tools such as Genetic Programming (Koza, 1992), these models allow the creation and evolution of novel agents, which are not constrained by pre-specified strategy types. However, this kind of models tends to focus on price dynamics, rather than fraction dynamics (Chen et al, 2010).

In a previous work (Chen et al, 2011), we combined properties from the $N$ type and autonomous models into a novel model. We first used Genetic Programming (GP) as a rule inference engine, which created and evolved autonomous agents; we then used Self-Organizing Maps (SOM) (Kohonen, 1982) as a clustering machine, and thus re-created the mesoscopic level that the $N$-type models represent, where agents were categorized into different strategy types. We then investigated the short- and long-term dynamics of the fractions of strategies that existed in a financial market. Nevertheless, that study rested upon an important assumption, i.e., the maps derived from each time period were comparable with each other. This comparability assumption itself required that the types (clusters), as well as their operational specification, would not change over time. If this were not the case, the subsequent study would be questioned. This was mainly due to one technical step in our analysis called translation. The purpose of translation was to place the behavior of agents observed in one period into a different period and to recluster it for the further cross-period comparison. We could not meaningfully have done this without something like topological equivalence, which could not be sustained without the constancy of the types.

However, this assumption can be considered as strict and unrealistic. Strategy types do not necessarily remain the same over time. For instance, if a chartist strategy type exists in time $t$, it is not certain it will also exist in $t+1$. If market conditions change dramatically, the agents might consider other strategy types as more effective and choose them. The chartist strategy would then stop existing.

In this paper, we relax the above assumption, since our current work does not require cross-period comparisons. Our model thus becomes more realistic. In addition, we shift our focus from fraction dynamics to behavior dynamics: we examine the plausibility of an observation made under artificial markets (Arthur, 1992), which suggests that the nature of financial markets constantly changes. This implies that trading strategies need to constantly co-evolve with the markets; if they do not, they become obsolete or dinosaurs (Arthur, 1992). We hence test if this observation holds in the 'real' world, under four empirical financial markets. This will offer important insights regarding the behavior dynamics of the markets.

The rest of this paper is organized as follows: Section 2 presents a brief overview of the different types of agent-based financial models that exist in the 
literature, and also discusses their limitations. In order to address these limitations, we created an agent-based financial model (Chen et al, 2011), which is presented in Sect. 3. More specifically, Sect. 3 presents the two techniques that our agent-based model uses, namely Genetic Programming and Self-Organizing Maps. In addition, Sect. 3 gives some background information on these two techniques, and also discusses the details of the algorithms we have used. Section 4 then presents the experimental designs, Sect. 5 reviews the testing methodology, and Sect. 6 presents the results of our experiments. Finally, Sect. 7 concludes this paper and discusses future work.

\section{Agent-based Financial Models}

Agent-based financial models are models of financial markets, where artificial agents can trade with each other. Building such models can give valuable information about different aspects of market dynamics, such as behavior dynamics (Chan et al, 1999). As we have already mentioned, Chen et al (2010) divides these models into two basic designs: the $N$-type design, and the autonomous agent design. The rest of this section presents these two designs.

\section{$2.1 \quad N$-type Designs}

Two- and Three-Type Designs In this type of designs, agents have beliefs regarding the price of a stock in the next period. In the two-type design, there are two types of agent beliefs. Consequently, there are two types of fixed and prespecified trading strategies. Each agent can only choose between these two types. These two types are usually the fundamentalists and the technical traders. ${ }^{3}$

The three-type design is an extension of the two-type one, where there are three types of agents. One way to implement this design is to have two types of chartists, the momentum traders and the contrarian traders (Sansone and Garofalo, 2007). The former is the kind of agents we described above as 'chartists'. The latter, the contrarian traders, extrapolate past movements of the price into the future, the opposite way that the trend goes. This happens because contrarians believe that the price trend will finish soon and will start to reverse.

Finally, we should mention that several extensions of the above designs exist, by enriching their behavioral rules. For instance, a typical way to do this is by adding a memory factor to these rules. Other extensions can be to add an adaptive behavior, where the agents can learn from their previous experiences. Such examples can be found in (Brock and Hommes, 1998), where Brock and Hommes use 2-, 3-, and 4-type models. Other adaptive behavior examples include Kirman's ANT Model (Kirman, 1991, 1993) and Lux's Interactive Agent Hypothesis Model (Lux, 1995, 1997, 1998).

\footnotetext{
${ }^{3}$ Other equivalent names for technical traders are chartists, trend-followers and noisy traders.
} 
Many-Type Designs So far we have seen designs with few 'fixed' types, namely two and three. However, in the literature we can find other $N$-type designs, where $N>3$.

\section{Adaptive Belief Systems}

A very good example of many-type design is the Adaptive Belief System (ABS) of Brock and Hommes (Brock and Hommes, 1998, 1997). This system can be considered as an extension of the two- and three-type designs we have seen. The number of strategies takes values from 1 to $N$ and these are known and fixed, like before. This means that agents can choose from a finite and fixed number of beliefs.

Large Type Limit and Continuous Belief Systems

Other many-type designs include the Large Type Limit (LTL) (Brock et al, 2005 ) and the the Continuous Belief System (CBS) (Dicks and Van der Weide, 2005). In these systems, the number $N$ of strategies is not finite, but infinite, i.e., $N \rightarrow \infty$. Both of these systems are based on an idea called distribution of beliefs, where there is a belief space from which the observed beliefs are sampled.

\subsection{Autonomous Agent Designs}

So far, we have talked about the $N$-type designs, where the trading strategies are pre-specified and fixed by the model designer. Thus, the agents are restricted in using these specific strategies and cannot come up with any new ones. Although the $N$-type design had been characterized as a major class of agent-based financial models, it has also been agreed that it severely restricts the degree of autonomy available for financial agents (Chen et al, 2010).

This issue of autonomy was addressed by the autonomous agent designs, where we can have artificial agents who are autonomous and thus have the ability of discovering new strategies, which have never been used before. An example of this is the well-known Santa Fe Institute (SFI) model (Palmer et al, 1994; Arthur et al, 1997), where a Genetic Algorith (GA) (Holland, 1975) was used. Thus, a fixed number of strategies does not exist; on the contrary, each artificial agent can have a different trading strategy which is "customized" by a GA. SFI is of course not the only application of GA in artificial stock markets. Another example is AGEDASI TOF ${ }^{4}$ (Izumi and Okatsu, 1996; Izumi and Ueda, 1999). If the reader is interested in these topics, a very good literature review can be found in (Chen et al, 2009). ${ }^{5}$

\footnotetext{
${ }^{4}$ It stands for A GEnetic-algorithmic Double Auction SImulation in TOkyo Foreign exchange market

${ }^{5}$ It should also be said that apart from GA, other population-based learning models have been used, such as GP. We refer the readers to (Chen et al, 2010) for more details.
} 


\subsection{Limitations of the Agent-Based Financial Models}

In the previous sections, we described the two main agent-based financial designs: the $N$-type and the SFI-like (autonomous agents design) ones. The former design consists of $N$ pre-specified strategy types, and the agents have to choose among these $N$ types. An advantage of this design is that it allows us to observe the changes in the market fraction ${ }^{6}$ dynamics of the above strategy types. However, as we saw, a disadvantage of this type of models is that the agents are restricted in choosing from the given $N$ strategy types. In addition, another limitation of this type of models is the lack of heterogeneity. Agents that belong in the same trading strategy type follow exactly the same behavioral rule. Nevertheless, in the real world, the behavior of each trader is expected to be heterogeneous; even if some traders are following a certain trading strategy type, it does not mean that the behave in exactly the same way.

As we saw, the issue of heterogeneity is addressed by the SFI-like models. This type of models allows the creation of autonomous and heterogeneous agents. Nevertheless, even under the autonomous agent models, agents have to choose among a pre-specified number $N$ of trading strategy types (Chen et al, 2010). To the best of our knowledge, there is no model that uses autonomous agents that are not restricted to predefined, fixed strategy types.

This thus motivated us to create such a model. In order to do this, we used Genetic Programming as a rule inference engine, and Self Organizing Maps as a clustering tool. The next section presents our model in detail.

\section{Model}

In this section, we present our agent-based financial model, which was first introduced in Chen et al (2011). This model first allows the creation of novel, autonomous and heterogeneous agents by the use of GP. The reason for using GP is because the market is considered as an evolutionary process; this is inspired by Andrew Lo's Adaptive Market Hypothesis (AMH) (Lo, 2004, 2005), where Lo argued that the principles of evolution (i.e., competition, adaptation, and natural selection) can be applied to financial interactions. Thus, agents can be considered to be organisms that learn and try to survive.

After creating and evolving novel agents, we cluster them into types of trading strategies via SOM. These types are thus not pre-specified, but depend on the strategies of the agents. In this way, we are able to reconstruct the microscopic level of markets (SFI-like designs), where financial agents are created, and connect it to the mesoscopic level ( $N$-type designs), where agents are clustered into $N$ strategy types. Figure 1 illustrates this process.

In the rest of Sect. 3 we give some brief background information on GP and SOM, explain how they were used in our agent-based model, and also present the algorithms we used behind these techniques.

\footnotetext{
${ }^{6}$ Market fraction refers to the proportion of the different trading strategy types (e.g., fundamentalists and chartists) that exist in a financial market.
} 


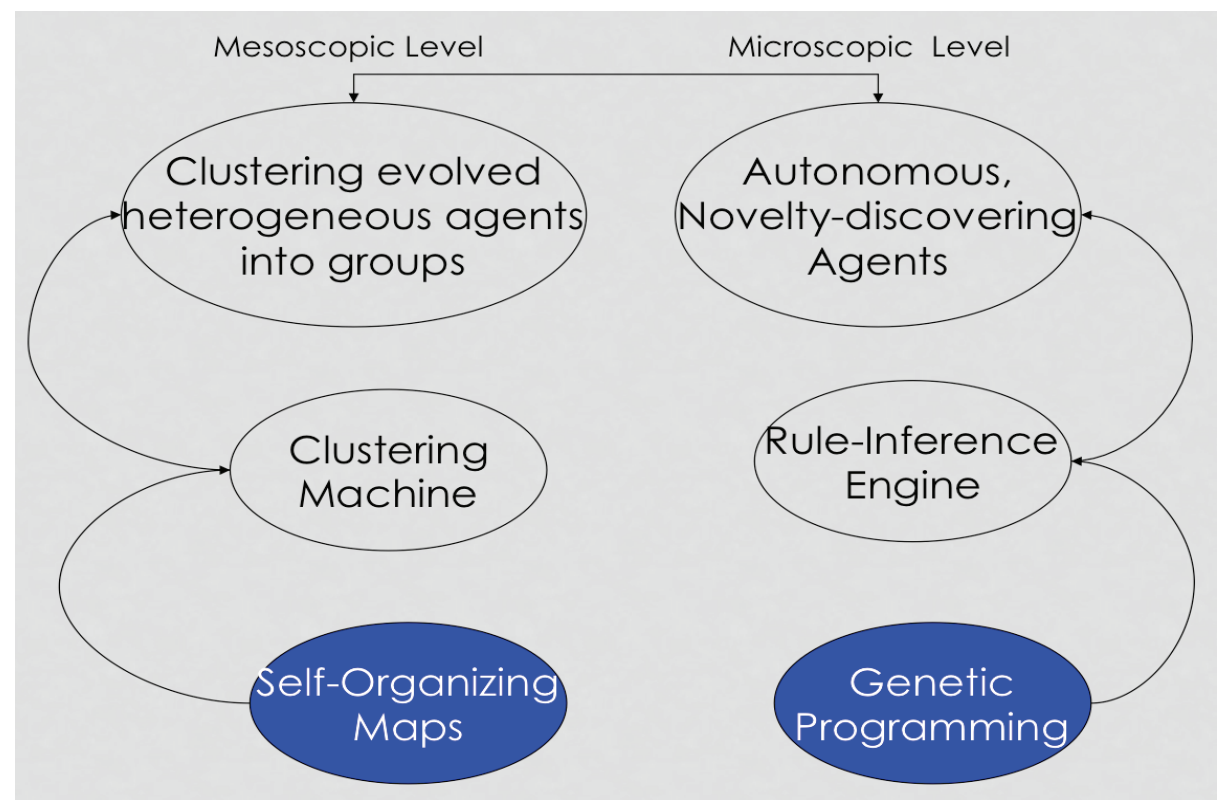

Fig. 1. Connecting the microscopic to the mesoscopic level of financial markets.

\subsection{Genetic Programming (GP)}

Genetic Programming (GP) (Koza, 1992, 1994; Koza et al, 1999, 2003; Banzhaf et al, 1998; Poli et al, 2008) is an evolutionary technique inspired by natural evolution, where computer programs act as the individuals of a population. The GP process has several steps. To begin with, a random population is created, by using terminals and functions appropriate to the problem domain, where the former are variables and constants of the programs, and the latter are responsible for processing the values of the system (either terminals or other functions' output).

In the next step, each individual is measured in terms of a pre-specified fitness function. The purpose of assigning a fitness to each individual is to measure how well it solves the problem. In the following step, individuals are chosen to produce new offspring programs. A typical way of doing this is by using the fitness-proportionate selection, where an individual's probability to be selected is equal to its normalized fitness value (Koza, 1992). The individuals chosen from the population are manipulated by genetic operators such as crossover and mutation, in order to produce offsprings. The new offsprings constitute the new population. Finally, each individual in the new population is again assigned a fitness and the whole process is repeated again, until a termination criterion is met (usually a number of generations). At the end of this procedure (last generation), the program with the highest fitness is considered as the result of that run. 
Next, we explain how GP was utilized in our model.

GP as a Rule-Inference Engine First of all, we assume that traders' behavior, including price expectations and trading strategies, is either not observable or not available. Instead, their behavioral rules have to be estimated by the observable market price. Using macro data to estimate micro behavior is not new, ${ }^{7}$ as many empirical agent-based models have already performed such estimations (Chen et al, 2010). However, such estimations are based on very strict assumptions, as we saw earlier (e.g., having pre-specified trading strategy types is considered to be a strict and unrealistic assumption). Since we no longer keep these assumptions, an alternative must be developed, and in this paper we recommend Genetic Programming (GP).

As we have already mentioned, the use of GP is motivated by considering the market as an evolutionary and selective process. ${ }^{8}$ In this process, traders with different behavioral rules participate in the markets. Those behavioral rules which help traders gain lucrative profits will attract more traders to imitate, and rules which result in losses will attract fewer traders. An advantage of GP is that it does not rest upon any pre-specified class of behavioral rules, like many other models in the agent-based finance literature (Chen et al, 2010). Instead, in GP, a population of behavioral rules is randomly initiated, and the survival-of-thefittest principle drives the entire population to become fitter and fitter in relation to the environment. In other words, given the non-trivial financial incentive from trading, traders are aggressively searching for the most profitable trading rules. Therefore, the rules that are outperformed will be replaced, and only those very competitive rules will be sustained in this highly competitive search process. ${ }^{9}$

Hence, even though we are not informed of the behavioral rules followed by traders at any specific time horizon, GP can help us infer what are the rules the traders follow, by simulating the evolution of the microstructure of the market. Traders can then be clustered based on realistic, and possibly complex behavioral rules. ${ }^{10}$ The GP algorithm used to infer the rules is presented in detail in the next section.

\footnotetext{
7 'Macro data' is generally a term used to mainly describe two categories of data: aggregated data, and system-level data Diez-Roux (2002). The former refers to data that combine information, such as unemployment statistics and demographics. The latter refers to information that cannot be disaggregated to lower level unities; such examples are the prices of a stock. On the other hand, 'micro behavior' refers to the study of the behavior of components of a national economy, such as individual firms, households and traders.

${ }^{8}$ See Lo $(2004,2005)$ for his eloquent presentation of the Adaptive Market Hypothesis.

${ }^{9}$ It does not mean that all types of traders surviving must be smart and sophisticated. They can be dumb, naive, randomly behaved or zero-intelligent. Obviously, the notion of rationality or bounded rationality applying here is ecological (Simon, 1956; Gigerenzer and Todd, 1999).

${ }^{10}$ Duffy and Engle-Warnick (2002) provides the first illustration of using genetic programming to infer the behavioral rules of human agents in the context of ultimatum game experiments.
} 
GP Algorithm Our GP is inspired by a financial forecasting tool, EDDIE (Kampouridis and Tsang, 2010), which applies genetic programming to evolve a population of market-timing ${ }^{11}$ strategies, which guide investors on when to buy or hold. These market timing strategies are formulated as decision trees, which, when combined with the use of GP, are referred to as Genetic Decision Trees (GDTs). Our GP uses indicators commonly used in technical analysis: Moving Average (MA), Trader Break Out (TBR), Filter (FLR), Volatility (Vol), Momentum (Mom), and Momentum Moving Average (MomMA). ${ }^{12}$ Each indicator has two different periods, a short- and a long-term one (12 and 50 days). Figure 2 presents a sample GDT generated by the GP. As we can observe, this tree suggests that the trader should buy if the 12 days Moving Average is less than 6.4. If, however, this is not the case, the tree examines the 50 days Momentum; if it is greater than 5.57, the then GDT recommends not-to-buy. If, finally, the 50 days Momentum is less than or equal to 5.57, then the GDT recommends to buy.

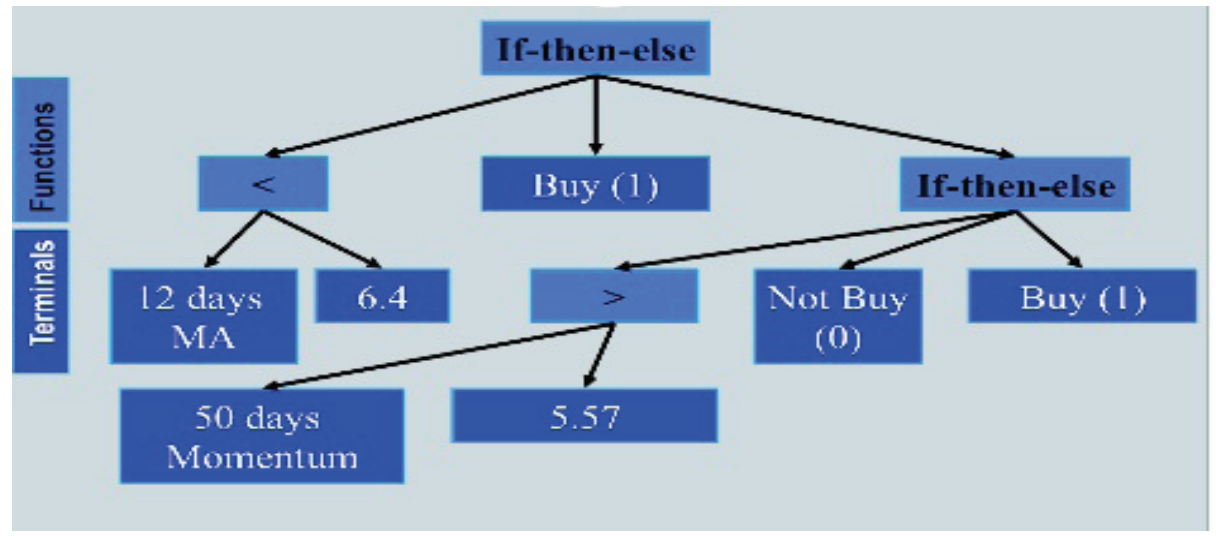

Fig. 2. Sample GDT generated by the GP.

Depending on the classification of the predictions, there are four cases: True Positive (TP), False Positive (FP), True Negative (TN), and False Negative $(\mathrm{FN})$. We then use the following 3 metrics, presented in Equations (1)-(3):

Rate of Correctness

$$
R C=\frac{T P+T N}{T P+T N+F P+F N}
$$

11 'Market timing' refers to the strategy of making buy or sell decisions of stocks, by attempting to predict future price movements.

${ }^{12}$ We use these indicators because they have been proved to be quite useful in previous works like Kampouridis and Tsang (2010). However, the purpose of this work is not to provide a list of the ultimate technical indicators. 
Rate of Missing Chances

$$
R M C=\frac{F N}{F N+T P}
$$

Rate of Failure

$$
R F=\frac{F P}{F P+T P}
$$

The above metrics combined give the following fitness function:

$$
f f=w_{1} * R C-w_{2} * R M C-w_{3} * R F
$$

where $w_{1}, w_{2}$ and $w_{3}$ are the weights for $\mathrm{RC}, \mathrm{RMC}$ and $\mathrm{RF}$, respectively, and are given in order to reflect the preferences of investors. For instance, a conservative investor would want to avoid failure; thus a higher weight for RF should be used. For our experiments, we chose to include GDTs that mainly focus on correctness and reduced failure. Thus these weights have been set to $1, \frac{1}{6}$ and $\frac{1}{2}$, respectively.

Given a set of historical data and the fitness function, GP is then applied to evolve the market-timing strategies in a standard way. After evolving a number of generations, what survives at the last generation is, presumably, a population of financial agents whose market-timing strategies are financially rather successful. We then use SOM to cluster these strategies into types of trading strategies.

\subsection{Self Organizing Maps (SOM)}

Self-Organizing Maps (SOM) (Kohonen, 1982) is a type of artificial neural networks which takes as input data with high dimensionality, ${ }^{13}$ and returns a lowdimensional representation of these data, along with their topological representation. This representation is called a map. A self-organizing map consists of components called neurons. Associated with each neuron is a weight vector, which has the same dimensions as the input data. During the SOM procedure, the weight vector of each neuron is dynamically adjusted via a competitive learning process. Eventually, each weight vector becomes the center (a.k.a. centroid) of a cluster of input vectors. Thus, at the end of the SOM procedure, all input vectors have been assigned to different clusters of a map.

The next section presents how SOM was applied to our model.

SOM as a Clustering Machine Once a population of rules is inferred from GP, it is desirable to cluster them based on a chosen similarity criterion. As we have already discussed at the beginning of Sect. 3 , this allows us to cluster heterogeneous agents into different types of trading strategies, which are neither fixed, nor pre-specified.

The similarity criterion which we choose is based on the observed trading behavior. ${ }^{14}$ Based on this criterion, two rules are similar if they are observationally

\footnotetext{
${ }^{13}$ In this work the input data is the market-timing vectors of the GDTs.

${ }^{14}$ Other similarity criteria could take place, too, such as risk averseness. However, in this paper we wanted to focus on the behavioral aspects of the rules.
} 
equivalent or similar, or, alternatively put, they are similar if they generate the same or similar market timing behavior. ${ }^{15}$

Given the criterion above, the behavior of each trading rule can be represented by its series of market timing decisions over the entire trading horizon, for example, 6 months. Therefore, when we denote the decision "buy" by "1" and "not-buy" by "0", then the behavior of each rule (GDT) is a binary vector. The dimensionality of these vectors is then determined by the length of the trading horizon. For example, if the trading horizon is 125 days long, then the dimension of the market timing vector is 125 . Thus, each GDT can be represented by a vector which contains a series of $1 \mathrm{~s}$ and 0 s, denoting the tree's recommendations to buy or not-buy on each day. Once each trading rule is concretized into its market timing vector, we can then easily cluster these rules by applying Kohonen's Self-Organizing Maps to the associated clusters.

The main advantage of SOMs over other clustering techniques such as Kmeans (MacQueen, 1967) is that the former can present the result in a visualizable manner, so that we can not only identify these types of traders, but also locate their 2-dimensional position on a map, i.e., a distribution of traders over a map. This provides us with a rather convenient grasp of the dynamics of the microstructure directly as if we were watching the population density on a map over time.

SOM Algorithm For our experiments, we use MathWorks' Neural Network toolbox $^{16}$, which is built in the MATLAB environment. We refer the reader to the relevant documentation for details on the algorithm.

Figure 3 presents a $3 \times 3 \mathrm{SOM}$ which has been produced by this toolbox. Here, 500 artificial traders are grouped into nine clusters (types of trading strategies). In a sense, this could be perceived as a snapshot of a nine-type agent-based financial market dynamics. Traders of the same type indicate that their market timing behavior is very similar. The market fraction or the size of each cluster can be seen from the number of traders belonging to that cluster. Thus, we can observe that the largest cluster has a market share of $71.2 \%(356 / 500)$, whereas the smallest one has a market share of $0.2 \%(1 / 500)$.

\section{Experimental Designs}

The experiments are conducted for a period of 17 years (1991-2007) and the data are taken from the daily closing prices of 4 international market indices: NYSE (USA), S\&P 500 (USA), STI (Singapore), and TAIEX (Taiwan). For statistical purposes, we repeat our experiments for 10 times.

$\overline{15}$ One might question the above similarity criterion, since very different rules might be able to produce the same signals. This does not pose a problem in this work, since we are interested in the behavior of the market (and thus the rules' behavior). We are not interested in the semantics aspect of the rules.

16 http://www.mathworks.com/access/helpdesk/help/toolbox/nnet/self_or4.html 


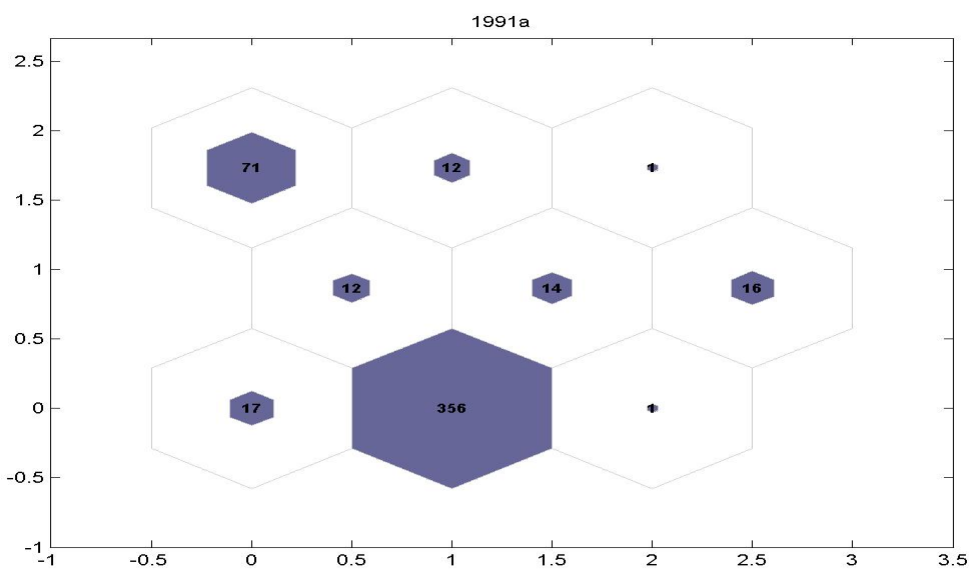

Fig. 3. Example of a $3 \times 3$ Self-Organizing Map

Each year is split into 2 halves (January-June, July-December), so in total, out of the 17 years, we have 34 periods. ${ }^{17}$ The first semester of a year is denoted with an 'a' at the end (e.g., 1991a), and the second semester of a year is denoted with a 'b' (e.g., 1991b). The GP systems is therefore executed 34 times, i.e., one time per period. Table 1 presents the GP parameters for our experiments. The GP parameters for our experiments are the ones used by Koza (Koza, 1992). Only the tournament size has been lowered, because we were observing premature convergence. Other than that, the results seem to be insensitive to these parameters. As we can observe, we have a population of 500 trading strategies. Thus, since there are 34 periods, we end up with 34 different populations of 500 strategies.

Table 1. GP Parameters

\begin{tabular}{lr}
\hline GP Parameters & \\
\hline Max Initial Depth & 6 \\
Max Depth & 17 \\
Generations & 50 \\
Population size & 500 \\
Tournament size & 2 \\
Reproduction probability & 0.1 \\
Crossover probability & 0.9 \\
Mutation probability & 0.01 \\
\end{tabular}

$\overline{17}$ At this point the length of the period is chosen arbitrarily as 6 months. We leave it to a future research to examine if and how this time horizon can affect our results. 
After generating and evolving strategies for each one of the 34 periods, we then use SOM to cluster these strategies into types. We do this for every one of the 34 periods. Thus, we end up with 34 different SOMs, one per semester, which represent the market in different time periods over the 17 -year horizon.

Table 2 presents the SOM parameters for our experiments. The parameters are the default ones provided in MATLAB's MathWorks Neural Network Toolbox.

Table 2. Default SOM parameters of the MathWorks SOM Toolbox.

\begin{tabular}{lr}
\hline SOM Parameters & \\
\hline Algorithm & Batch \\
Distance & Euclidean \\
Neighborhood Radius & $\sigma=3$ \\
Topology & Hexagonal \\
Steps & 100 \\
\hline
\end{tabular}

Finally, we define as 'base period', the period during which GP creates and evolves GDTs. We also define 'future period(s)', as the period(s) which follow(s) the base period (in chronological order).

\section{$5 \quad$ Testing Methodology}

As we mentioned at the beginning of this paper, we are interested in investigating Arthur's observation about the constantly changing behavior of financial markets (Arthur, 1992). In order to investigate whether the behavior of markets is nonstationary, we recluster the GDTs of each base period, to all future periods' clusters. ${ }^{18}$ By applying the same GDTs (strategies) to clusters of future periods, we can observe how well these strategies fit in the new environments (clusters). The logic behind this is the following: when we first evolved and clustered the GDTs (base period), these GDTs were placed in clusters that represented their respective strategies. For instance, if there was a strategy type (cluster) that represented 'chartists', then all GDTs which followed a chartist strategy were placed in that cluster. When we then take the GDTs from a base period and recluster them to strategy types of future periods, it is not guaranteed that there will again be a cluster that represents chartists. If the market constantly changes, there is a possibility that this type of strategies does not exist any more in the future periods. Thus, the GDTs find themselves unadapted to the new environment (clusters) and have to choose another cluster, which represents them as closely as possible. This cluster will be the one that has the centroid with the

\footnotetext{
$\overline{18 \text { The process }}$ of reclustering is explained later in this section.
} 
smallest Euclidean distance ${ }^{19}$ from the market-timing vectors of these GDTs. Of course, since now the SOM of the future period is formed by different clusters, the GDTs might not fit in as well as they did in the base period. In order to measure this 'unfitting', we use a dissatisfaction rate, i.e., how dissatisfied these GDTs will be when placed into a future period's cluster that does not represent their strategy. If the market is non-stationary, the GDTs' dissatisfaction rate will be high, as a result of the changes that took place in the market. The dissatisfaction rate is defined as the Euclidean distance of a GDT's market-timing vector to the centroid of the cluster in which it is placed, after the reclustering procedure. Under a non-stationary market behavior, the following statement should hold:

The average dissatisfaction rate of the population of GDTs from future periods should not return to the range of dissatisfaction of the base period.

Hence, we will test the above statement against the 4 financial markets.

Let us now explain the process of reclustering. We start with 1991a as the base period. Each evolved GDT is moved to the next period, 1991b, and reclustered into one of the clusters of that period. In order to 'decide' which cluster to choose, the GDT compares the Euclidean distance of its market timing vector to the centroid of each cluster; it is then placed into the cluster with the smallest Euclidean distance. The same procedure follows for all GDTs of the population. At the end, the population of evolved GDTs from the base period of 1991a will have been reclustered into the clusters of period 1991b. The same procedure is followed in all future periods. This means that the GDTs from 1991a are also reclustered into 1992a, 1992b, ..., 2007b. Finally, the same process is done for all other base periods (i.e., 1991b, 1992a, ..., 2007a).

Once the process of reclustering is complete, we calculate the dissatisfaction rate of each GDT in the population. Next, we calculate the population's average dissatisfaction rate. We do the same for all 34 periods. Given a base period, the population average dissatisfaction of all periods is normalized by dividing those population average dissatisfaction rates by the population average dissatisfaction rate in the base period. For instance, if the population dissatisfaction rates for periods 1991a, 1991b, 1992a, ..., 2007b are 0.8, 0.85, 0.9, ..., 0.85, respectively, the the normalized population dissatisfaction for the base period 1991a would be $\frac{0.8}{0.8}, \frac{0.85}{0.8}, \frac{0.9}{0.8}, \ldots, \frac{0.85}{0.8}$. Hence, each base period has its normalized average dissatisfaction rate equal to 1 . In order to prove that the market is non-stationary, we need to show that the normalized average dissatisfaction rate of the GDTs increases in the future periods, and never returns to its initial value of 1 , which was during the base period. If, on the other hand, this rate reaches 1 or below, it is an indication of a cyclic market behavior, since the GDTs have found the same conditions with the base period, and as a result feel as 'satisfied' as before.

Finally, we define as dinosaurs the population of GDTs that has been reclustered from a base period to future periods. The reason of calling them in this

\footnotetext{
${ }^{19}$ One may wonder if the choice of the Euclidean distance as a distance metric, when the vectors of the GDTs are binary, is an appropriate one. However, this does not pose a problem, because the vectors of the clusters' centroids are real valued.
} 
way is because these GDTs have not adapted to the new market environment (clusters of the SOMs from future periods) and are thus ineffective. If these GDTs' normalized average dissatisfaction rate drops to less than or equal to 1, we call them returning dinosaurs, because they have become effective again. ${ }^{20}$

\section{Results}

As explained, returning dinosaurs denote a cyclic market behavior. To examine if dinosaurs return, we iterate through each base period and calculate the minimum normalized average dissatisfaction rate for each future period. This gives us an indication of how many returning dinosaurs, if any, exist. If, for instance, 1991a is the base period, then there is a series of 33 population dissatisfaction values for its future periods. We obtain the minimum value among these 33 values, in order to check how close to 1 the normalized dissatisfaction rate of this future period is. This process is then repeated for 1991b as the base period and its 32 future periods, and so on, until base period 2007a. We thus end up with a $1 \times 33$ vector, which presents the minimum dissatisfaction per base period and thus shows whether any returning dinosaurs exist. In addition, we are interested in investigating whether different number of clusters (strategy types) can affect the test results. We thus run tests under 2 to 9 clusters, for the following SOM dimensions: $2 \times 1,3 \times 1,2 \times 2,5 \times 1,3 \times 2,7 \times 1,4 \times 2$, and $3 \times 3$. Figure 4 presents the graphs of the minimum dissatisfaction vectors for the 4 international markets. Each line represents the results of a different SOM dimension. The horizontal line indicates a dissatisfaction of 1 , and is given as a reference. Results are on average of 10 runs.

What we can see from Fig. 4 is that there are no base periods with a minimum normalized dissatisfaction rate below 1 . This observation holds under all SOM dimensions, and under all of the financial markets tested in this paper. In fact, the closest to 1 that the dissatisfaction rate gets is around 2, for S\&P 500 (1999a), STI (1998a), and TAIEX (1999b). But even these periods could be treated as exceptional cases, because it is obvious that the average dissatisfaction rate is much higher than 2 .

To make the above argument even clearer, we present the average dissatisfaction rates over the 10 runs, at Table 3. Each row presents the average dissatisfaction rate for a different market, and each column presents the rate under a different SOM dimension. As we can observe, these values range from 4.44 $($ NYSE- $2 \times 1$ SOM) to $8.88($ STI- $3 \times 3 \mathrm{SOM})$. In addition, the average dissatisfaction rate ranges from 4.75 (2 clusters) to 7.85 (9 clusters). It is thus obvious that on average, no dinosaurs return, because the average dissatisfaction rate

${ }^{20}$ In a previous work (Kampouridis et al, 2010), where we investigated the markets' behavior dynamics by only using GP but not SOM, we did not use this 'strict' definition of returning dinosaurs. This led us to conclude that returning dinosaurs existed. However, if we had also used the current paper's definition, the results from (Kampouridis et al, 2010) would not have dramatically differed from the current paper. 

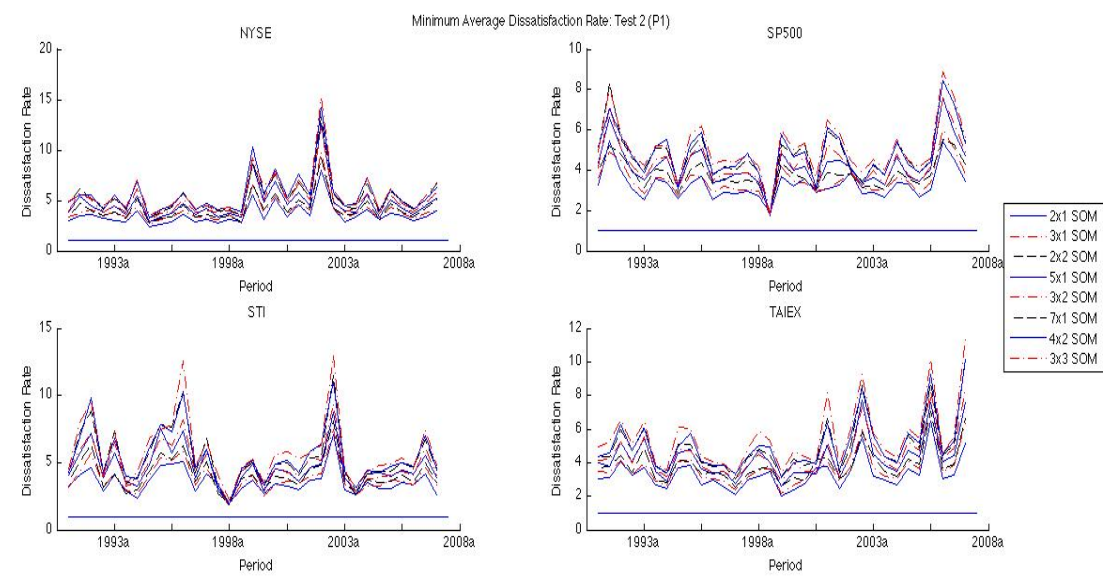

Fig. 4. Minimum normalized dissatisfaction rate of the population of GDTs per base period, for all SOM dimensions, for all datasets. Each subfigure represents a single dataset. From left to right, top to bottom: NYSE, S\&P 500, STI, TAIEX.

is much higher than 1. But even if we want to take into account the outliers (minimum dissatisfaction rate), not many things change. Table 4 presents the average, over the 10 runs, of the minimum dissatisfaction rates. As we can see, these rates range from $3.28(\mathrm{~S} \& \mathrm{P} 500-2 \times 1 \mathrm{SOM})$ to $5.79($ STI- $3 \times 3 \mathrm{SOM})$. Furthermore, the average of the minimum dissatisfaction rate per SOM dimension is 3.41 for the $2 \times 1 \mathrm{SOM}$, and it gradually increases, as the number of clusters increases, reaching 5.46 for the $3 \times 3$ SOM. Hence, the minimum dissatisfaction rate is on average quite far away from 1 , which as we mentioned is the threshold for a returning dinosaur.

Table 3. Average of Average Dissatisfaction Rate per Cluster per Dataset

\begin{tabular}{cclllllll}
\hline & $2 \times 1$ & $3 \times 1$ & $2 \times 2$ & $5 \times 1$ & $3 \times 2$ & $7 \times 1$ & $4 \times 2$ & $3 \times 3$ \\
\hline NYSE & 4.44 & 4.90 & 5.22 & 5.92 & 6.17 & 6.88 & 6.96 & 7.27 \\
S\&P500 & 4.36 & 4.61 & 4.93 & 5.53 & 5.79 & 6.45 & 6.56 & 6.86 \\
STI & 5.17 & 5.65 & 6.11 & 6.98 & 7.19 & 8.30 & 8.33 & 8.88 \\
TAIEX & 5.04 & 5.48 & 5.74 & 6.54 & 6.96 & 7.76 & 7.78 & 8.40 \\
& & & & & & & & \\
\hline Mean & 4.75 & 5.16 & 5.50 & 6.24 & 6.53 & 7.35 & 7.41 & 7.85 \\
\hline
\end{tabular}

An observation we can make from the above results is that the trading strategies never reach a dissatisfaction rate of 1 . Thus, returning dinosaurs do not exist. Market conditions constantly change and the strategies cannot be as "satisfied" as they were in the base period. However, there can be a few exceptions, where 
Table 4. Average of Minimum Dissatisfaction Rate per Cluster per Dataset

\begin{tabular}{ccccccccc}
\hline & $2 \times 1$ & $3 \times 1$ & $2 \times 2$ & $5 \times 1$ & $3 \times 2$ & $7 \times 1$ & $4 \times 2$ & $3 \times 3$ \\
\hline NYSE & 3.52 & 3.94 & 4.14 & 4.69 & 4.98 & 5.33 & 5.53 & 5.56 \\
S\&P500 & 3.28 & 3.48 & 3.77 & 4.18 & 4.31 & 4.78 & 4.80 & 5.07 \\
STI & 3.56 & 3.83 & 4.09 & 4.61 & 4.79 & 5.34 & 5.41 & 5.79 \\
TAIEX & 3.29 & 3.64 & 3.83 & 4.25 & 4.44 & 4.88 & 5.00 & 5.43 \\
& & & & & & & & \\
\hline Mean & 3.41 & 3.72 & 3.96 & 4.43 & 4.63 & 5.08 & 5.19 & 5.46 \\
\hline
\end{tabular}

the dissatisfaction rate goes quite low, e.g. around 2. Although we cannot say that dinosaurs have returned, it is obvious that the market in those years has similarities to the base period. In these cases, we can say that dinosaurs have returned as lizards. Nevertheless, strategies that have not co-evolved with the market, cannot reach performance levels as the ones they once had in their base period (i.e., no returning dinosaurs). Market conditions change continuously.

The above observations are very important and allow us to argue that the behavior of the 4 markets tested in this paper constantly changes. Trading strategies need to adapt to these changes, in order to remain effective. If they do not, they find the new environment (clusters) very different from the one in the base period and thus are very "dissatisfied". These strategies thus become obsolete or dinosaurs.

One final observation we can make is that the number of clusters does not affect the test's results. The dissatisfaction rate of each market follows always the same pattern, regardless the number of clusters. No returning dinosaurs are observed, under any number of the trading strategy types tested.

\section{Conclusion}

To conclude, this paper presented a significant extension to a previous market microstructure model (Chen et al, 2011), and also discussed preliminary results on the behavior dynamics of financial markets.

In Chen et al (2011), we used Genetic Programming (GP) as a rule-inference engine to find out the behavioral rules of agents, and Self-Organizing Maps (SOM) to cluster these agents. However, because of an important assumption in that work, we had to require that SOM clusters, as well as their operational specification, would remain the same over time. In this paper, we relaxed that assumption. This offered more realism to our model and allowed us to investigate market behavior dynamics.

Our experimental work was inspired by an an observation made under artificial agent-based financial markets (Arthur, 1992). This observation says that the nature and constituents of agents, and thus their strategies, constantly change; if these strategies do not continuously adapt to the changes in their environments, 
then they become obsolete (dinosaurs). In order to test the plausibility of this observation, we ran tests on 4 international financial markets.

The results showed that on average, these datasets did not demonstrate the existence of returning dinosaurs, and thus verified the existence of the nonstationary property in financial markets' behavior. The implications of this are very important. Strategies from the past cannot be successfully re-applied to future periods, unless they have co-evolved with the market. If they have not, they become obsolete, because the market conditions change continuously. They can occasionally return as lizards, meaning that these strategies can sometimes demonstrate relatively good performance, but they cannot become again as successful, as they once were.

Future work will focus on exploring whether the above observations can hold under even more financial markets. In addition, we aim to show that our experimental results are independent to the GP and SOM algorithms we have used in this paper. We have already done some work towards this direction, where we ran experiments under 2 different GP algorithms and found that results were independent to the choice of the algorithm (Kampouridis et al, 2011). Moreover, we are also interested in demonstrating that our results would be the same if we were using different rule inference engines and clustering machines. To show this, we aim to run experiments where we use Genetic Algorithm instead of Genetic Programming and other clustering techniques, such as standard hierarchical clustering (Xu and Wunsch, 2008) and growing hierarchical SOMs (Dittenbach et al, 2001), instead of the classical SOM approach.

\section{Acknowledgments}

This work was supported by an EPSRC grant (No. EP/P563361/01) and by AI-Econ Center of National Cheng Chi University, Taiwan. 


\section{Bibliography}

Arthur B (1992) On learning and adaptation in the economy, working paper 92-07-038, Santa Fe Institute

Arthur W, Holland J, LeBaron B, Palmer R, Tayler P (1997) Asset pricing under endogenous expectations in an artificial stock market. In: Arthur B, Durlauf S, Lane DE (eds) The Economy as an Evolving Complex System II, Addison-Wesley, Reading, MA, pp 15-44

Banzhaf W, Nordina P, Keller R, Francone F (1998) Genetic Programming: An Introduction: On the Automatic Evolution of Computer Programs and its Applications. Heidelberg and Morgan Kaufmann

Brock W, Hommes C (1997) A rational route to randomness. Econometrica 65:1059-1095

Brock W, Hommes C (1998) Heterogeneous beliefs and routes to chaos in a simple asset pricing model. Journal of Economic Dynamics and Control 22:12351274

Brock W, Hommes C, Wagener F (2005) Evolutionary dynamics in markets with many trader types. Journal of Mathematical Economics 41:7-42

Chan N, LeBaron B, Lo A, Poggio T (1999) Agent-based models of financial markets: A comparison with experimental markets. MIT Artificial Markets Project Paper No. 124

Chen SH, Huang YC, Wang JF (2009) Bounded rationality and the elasticity puzzle: An analysis of agent-based computational consumption capital asset pricing models. In: Zambelli, S. (ed.), Routledge

Chen SH, Chang CL, Du YR (2010) Agent-based economic models and econometrics. Journal of Knowledge Engineering Review (forthcoming)

Chen SH, Kampouridis M, Tsang E (2011) Microstructure dynamics and agentbased financial markets. In: Bosse T, Geller A, Jonker CM (eds) Multi-AgentBased Simulation XI, 11th International Workshop, Revised Papers, LNAI, Springer-Verlag, Berlin Heidelberg, vol 6532, pp 121-135, forthcoming

Dicks C, Van der Weide R (2005) Herding asynchronous updating and heterogeneity in memory in a CBS. Journal of Economic Dynamics and Control 29 (4):741-763

Diez-Roux A (2002) A glossary for multilevel analysis. Journal of Epidemiology and Community Health 56:588-594

Dittenbach M, Rauber A, Merkl D (2001) Recent advances with the growing hierarchical self-organizing map. In: Allinson N, Yin H, Allinson L, Slack J (eds) Proceedings of the 3rd Workshop on Self-Organizing Maps, Springer, Lincoln, England, Advances in Self-Organizing Maps, pp 140-145

Duffy J, Engle-Warnick J (2002) Using symbolic regression to infer strategies from experimental data, Springer, pp 61-82. Evolutionary Computation in Economics and Finance 
Gigerenzer G, Todd P (1999) Fast and Frugal Heuristics: The Adaptive Toolbox, Oxford University Press, pp 3-34. Gigerenzer, G. Todd, P. and the ABC Research Group

Holland J (1975) Adaptation in Natural and Artificial Systems. University of Michigan Press, Ann Arbor, MI

Izumi K, Okatsu T (1996) An artificial market analysis of exchange rate dynamics. In: Fogel L J BTE Angeline P J (ed) Evolutionary Programming V, MIT Press, pp 27-36

Izumi K, Ueda K (1999) Analysis of dealers' processing financial news based on an artificial market approach. Journal of Computational Intelligence in Finance 7:23-33

Kampouridis M, Tsang E (2010) EDDIE for investment opportunities forecasting: Extending the search space of the GP. In: Proceedings of the IEEE Conference on Evolutionary Computation, Barcelona, Spain, pp 2019-2026

Kampouridis M, Chen SH, Tsang E (2010) Testing the dinosaur hypothesis under empirical datasets. In: Schaefer R, Cotta C, Kołodziej J, Rudolph G (eds) Parallel Problem Solving from Nature - PPSN XI, Springer, Lecture Notes in Computer Science, vol 6239, pp 199-208

Kampouridis M, Chen SH, Tsang E (2011) Investigating the effect of different GP algorithms on the non-stationary behavior of financial markets. In: Computational Intelligence for Financial Engineering and Economics, IEEE Press, IEEE Symposium Series on Computational Intelligence, p forthcoming

Kirman A (1991) Epidemics of Opinion and Speculative Bubbles in Financial Markets, M. Taylor (Hrsg.), "Money and Financial Markets", London, Macmillan, pp 354-368

Kirman A (1993) Ants, rationality and recruitment. Quarterly Journal of Economics 108(1):137-156

Kohonen T (1982) Self-organized formation of topologically correct feature maps. Journal of Biological Cybernetics 43:59-69

Koza J (1992) Genetic Programming: On the programming of computers by means of natural selection. Cambridge, MA: MIT Press

Koza J (1994) Genetic Programming II: Automatic Discovery of Reusable Programs. Cambridge, MA: MIT Press

Koza J, Andre D, Bennett III F, Keane M (1999) GeneticProgramming 3: Darwinian Invention and Problem Solving. Morgan Kaufman

Koza J, Keane M, Streeter M, Mydlowec W, Yu J, Lanza G (2003) Genetic Programming IV: Routine Human-Competitive Machine Intelligence. Kluwer Academic Publishers

Lo A (2004) The adaptive market hypothesis: market efficiency from an evolutionary perspective. Journal of Portfolio Management 30:15-29

Lo A (2005) Reconciling efficient markets with behavioral finance: The adaptive markets hypothesis. Journal of Investment Consulting 2:21-44

Lux T (1995) Herd behavior, bubbles and crashes. Economic Journal 105:880896

Lux T (1997) Time variation of second moments from a noise trader/infection model. Journal of Economic Dynamics and Control 22:1-38 
Lux T (1998) The socio-economic dynamics of speculative markets: Interacting agents, chaos and the fat tails of return distributions. Journal of Economic Behavior and Organization 33:143-165

MacQueen JB (1967) Some methods for classification and analysis of multivariate observations. In: Proceedings of the 5th Berkeley Symposium on Mathematical Statistics and Probability, University of California Press, pp 281-297

Palmer R, Arthur W, Holland J, LeBaron B, Tayler P (1994) Artificial economic life: a simple model of a stock market. Physica D 75:264-274

Poli R, Langdon W, McPhee N (2008) A Field Guide to Genetic Programming. Lulu.com

Sansone A, Garofalo G (2007) Asset price dynamics in a financial market with heterogeneous trading strategies and time delays. Physica A 382:247-257

Simon H (1956) Rational choice and the structure of environments. Psychological Review 63:129-138

Xu R, Wunsch D (2008) Clustering. Wiley-IEEE Press 